\title{
Serum Inhibitor of C5 Fragment-Mediated Polymorphonuclear Leukocyte Chemotaxis Associated with Chronic Hemodialysis
}

\author{
Simeon E. Goldblum, Dennis E. Van Epps, and William P. ReEd, Departments of \\ Medicine and Microbiology, Veterans Administration Hospital and University of \\ New Mexico School of Medicine, Albuquerque, New Mexico 87108
}

\begin{abstract}
A B S T RAC T Abnormal granulocyte chemotaxis has been described in chronic hemodialysis patients. In this study, sera from 53 hemodialysis patients were tested for chemotactic inhibitory activity by a modified Boyden technique. Chemotactic inhibitory activity, defined as $>20 \%$ inhibition of normal granulocyte chemotaxis, was found in $45 \%$ of patients. Only sera from patients having undergone $>3$ mo hemodialysis displayed chemotactic inhibitory activity and retained this inhibitory activity when retested 9 mo later. Four of five patients who had initially undergone $<3$ mo hemodialysis and lacked serum chemotactic inhibitory activity developed inhibitory activity when tested $9 \mathrm{mo}$ later. Clinical evaluation of patients with serum chemotactic inhibitory activity showed that these patients did not have a significantly increased incidence of infection, although a trend toward decreased mortality during the time of study was observed $(P=0.0721)$.

Serum chemotactic inhibitory activity was heat stable at $56^{\circ} \mathrm{C}$ for $30 \mathrm{~min}$ and concentration dependent. The major inhibitory component was found to have a sedimentation coefficient of $4 \mathrm{~S}$ by sucrose density gradient centrifugation. The chemotactic inhibitory activity was not precipitated by $30 \%$ ammonium sulfate, but was partially precipitated by $50 \%$ ammonium sulfate.
\end{abstract}

Inhibitory sera effectively suppressed neutrophil migration in response to chemotactic C5 fragment and Escherichia coli derived chemotactic factor but was least effective in a system mediated by casein. Furthermore, normal neutrophils preincubated in hemodialysis patient sera displayed normal chemotactic responsiveness indicating a lack of cell-directed inhibition. Serum fractions that contained the inhibitor were found to

\footnotetext{
Address requests for reprints to Dr. Van Epps.

Received for publication 9 June 1978 and in revised form 23 February 1979.
}

directly act on the chemotactic C5 fragment, reducing its chemotactic activity. This study indicates that a circulating $4 \mathrm{~S}$, heat-stable, factor-directed inhibitor of granulocyte chemotaxis is present in the sera of many hemodialysis patients and probably results from the hemodialysis procedure.

\section{INTRODUCTION}

An increased incidence of infectious complications has been associated with both nondialyzed chronic renal insufficiency $(1,2)$ and chronic hemodialysis (HD) ${ }^{1}$ $(3,4)$. Granulocyte abnormalities involving phagocytosis (5-7), in vitro random mobility (8), granulocyte adherence $(9,10)$, and chemotaxis (11-15) have all been reported in these patients. Chemotactic abnormalities have included suppressed cellular responsiveness (12-15) and diminished ability to generate chemotactic factors in the serum $(11,12)$. In 1975, Baum et al. (13) reported that up to $40 \%$ of nondialyzed uremic patients had a polymorphonuclear leukocyte (PMN) cellular chemotactic defect that could be improved with from 3 wk to 3 mo of maintenance HD. Cells from 6 to $15(40 \%)$ uremic patients had subnormal chemotactic indices, whereas those from 22 HD patients fell within the normal range. The chemotactic defect was not temporally related to the HD procedure. Generation of endotoxin-induced chemotactic factors and hemolytic complement levels were normal in the sera of the HD patients. In 1976, Salent et al. (12) reported depressed cellular PMN chemotaxis in 8 of $11 \mathrm{HD}$ patients as well as decreased chemotactic activity in endotoxin-activated sera in 9 of 11 patients. These defects did not parallel increased serum levels

\footnotetext{
${ }^{1}$ Abbreviations used in this paper: CD, complementderived, CIA, chemotactic inhibitory activity; HBSS, Hanks' balanced salt solution; HD, hemodialysis; HPF, high-power field, PMN, polymorphonuclear leukocyte(s).
} 
of urea nitrogen or creatinine nor were they affected by underlying etiologies of renal disease. Studies by Greene et al. (14) and Bjorksten et al. (15) showed further depression of $\mathrm{PMN}$ chemotactic responsiveness after HD treatments, without the presence of a circulating plasma inhibitor. All four of these recent studies have reported an intrinsic PMN chemotactic defect in a portion of HD patients (12-15), whereas only one study has demonstrated a serum defect (12). In the present study, we present evidence for serum chemotactic inhibitory activity (CIA) in HD patients. Furthermore, serum CIA may result from the HD procedure itself.

\section{METHODS}

Subjects. Serum was obtained from 53 patients undergoing HD. Samples of whole blood were obtained from a peripheral vein or through HD tubing immediately before $H D$, before any heparin had been introduced. All sera were rapidly separated from clotted specimens and stored at $-70^{\circ} \mathrm{C}$. Sera from nine of these patients were obtained immediately before and $4 \mathrm{~h}$ after HD.

Cell preparations. PMN were prepared from heparinized whole blood ( $0.01 \mathrm{U}$ heparin $/ 1.0 \mathrm{ml}$ whole blood) by Plasmagel (HTI Corp., Associate Biomedic Systems, Inc., Buffalo, N. Y.) erythrocyte sedimentation $(0.2 \mathrm{ml}$ Plasmagel $/ 1.0 \mathrm{ml}$ whole blood) and Ficoll-Hypaque gradient centrifugation (Ficoll, Pharmacia Fine Chemicals, Piscataway, N. J.; Hypaque, Winthrop Laboratories, New York) (16). The cell pellet that contained granulocytes was subsequently washed and resuspended in Hanks' balanced salt solution (HBSS) to a concentration of $7.0 \times 10^{6} \mathrm{cells} / \mathrm{ml}$. Cell preparations in our study were $>95 \%$ PMN.

Chemotactic factors. Fresh serum was incubated overnight at $4^{\circ} \mathrm{C}$ with $1 \mathrm{M}$ epsilon amino caproic acid. The following day, serum complement was activated with baker's yeast to generate the low molecular weight chemotactic C5 fragment according to the method of Vallota and MullerEberhard (17). This preparation was subsequently passed though a PM30 membrane (Amicon Corp, Lexington, Mass.), which excluded molecules with a mol wt of $>30,000$, and allowed passage of chemotactic C5 fragment. This preparation, referred to as complement-derived (CD) chemotactic factor, was then titrated in the chemotaxis assay as described below to give $\cong 200 \mathrm{PMN} / \mathrm{high}$ power field. The chemotactic activity of this preparation was $98 \%$ inhibited by anti-human C5.

A $20 \%$ saturated solution of casein in minimum essential medium with Eagle's salts or a $10 \%$ solution of bacterial derived chemotactic factor were also used to stimulate chemotaxis (18). Bacterial factor was prepared by culturing Escherichia coli K12 bacteria in minimum essential medium with Eagle's salts for $24 \mathrm{~h}$ followed by removal of bacteria by filtration.

Chemotaxis assay. A modification of the Boyden technique with $5-\mu \mathrm{m}$ pore size membranes (Sartorius Filters, Inc., S. San Francisco, Calif.) to separate the upper cell suspension compartment and the lower chemotactic factor compartment was used to assess $P M N$ chemotaxis (16). The PMN suspension, $0.4 \mathrm{ml}$ at $7.0 \times 10^{6} \mathrm{PMN} / \mathrm{ml}$, was placed in the upper compartment and chemotactic factor plus a $10 \%$ serum test sample in the lower $0.25 \mathrm{ml}$ compartment. Chemotaxis was allowed to proceed for $2 \mathrm{~h}$ at $37^{\circ} \mathrm{C}$, after which membranes were fixed in formaldehyde, stained with hemotoxylin, and mounted on microscope slides. The number of PMN migrating to the distal side of the membrane was counted in five $\times 400$ high-power fields/membrane and averaged. Each sample was run in duplicate. In those assays testing pre- and post-HD sera from the same patient, the same donor cells were used.

In the PMN chemotaxis assay, $0.1 \mathrm{ml}$ of test sera was added to $0.9 \mathrm{ml}$ of the CD chemotactic factor-HBSS solution. This solution was placed in the $0.25 \mathrm{ml}$ lower compartment of the chemotaxis chamber. In controls that lacked test sera, only the CD chemotactic factor solution was used in the lower chamber. The effect of test sera on the system was expressed relative to this simultaneously run control. The mean number of PMN per high-power field (HPF) \pm 1 SD for the control values with $C D$ chemotactic factor was $189.7 \pm 90$. Values for each control and test sample had to be within $20 \%$ of the mean of the duplicate membranes for the assay to be considered valid.

Ammonium sulfate treatment. Test serum samples were salted out at varying percentages of ammonium sulfate $\left(\mathrm{NH}_{4} \mathrm{SO}_{4}\right)$ and centrifuged at $10,000 \mathrm{rpm}$ for $10 \mathrm{~min}$. Supernates were collected and precipiates were washed with the appropriate concentration of $\mathrm{NH}_{4} \mathrm{SO}_{4}$ and resuspended to original volume in phosphate-buffered saline. These samples were then dialyzed for $24 \mathrm{~h}$ at $4^{\circ} \mathrm{C}$ against multiple changes of phosphate-buffered saline. The soluble and precipitating fractions were tested for CIA.

Sucrose density gradient centrifugation. Sucrose density gradients $(5-20 \%, 4.8 \mathrm{ml})$ were used to separate whole serum from HD patients (16). In each case $0.2 \mathrm{ml}$ of a $1: 3$ dilution of whole serum was layered onto the gradients, which were then centrifuged at $100,000 \mathrm{~g}$ for $16 \mathrm{~h}$ in a Beckman L-2B ultra-centrifuge (Beckman Instruments, Inc., Fullerton, Calif.). Gradients were tapped from the bottom and divided into 15 fractions. Simultaneous gradients were run with ovalbumin and human immunoglobulin (Ig)G as markers. Fractions were dialyzed against HBSS and tested for CIA. In each case, chemotactic factor was added directly to the fraction, incubated at $37^{\circ} \mathrm{C}$ for $15 \mathrm{~min}$, and placed in the lower compartment of the chemotactic chamber.

\section{RESULTS}

\section{CIA in HD patient serum}

Sera from $53 \mathrm{HD}$ patients and 10 normals were tested for CIA with normal adult PMN as indicator cells (Fig. 1A). Chemotactic responses to test sera were expressed as the percent inhibition when compared to responses obtained with the control CD chemotactic factor alone. $20 \%$ or greater inhibition was considered meaningful in this assay system because laboratory controls under similar conditions with 30 normal sera never suppressed CD chemotactic fragment-mediated chemotaxis by $>20 \%$ (mean inhibition, $1.5 \pm 5 \%$ ). 24 $(45 \%)$ of the HD patient sera displayed $>20 \%$ inhibition of PMN chemotaxis, with nine $(17 \%)$ showing greater than 50\% inhibition. Tests with 10 normal sera showed no CIA. Although a subpopulation of HD patients existed in which no inhibition was observed, the mean inhibition of the total HD patient population (CIA, $21 \%$ ) was significantly greater than for normal subjects $(P<0.005$ by the Mann-Whitney $U$ test). Excess quantities of chemotactic factor in patients' sera could potentially "deactivate" PMN, resulting in a suppressed 


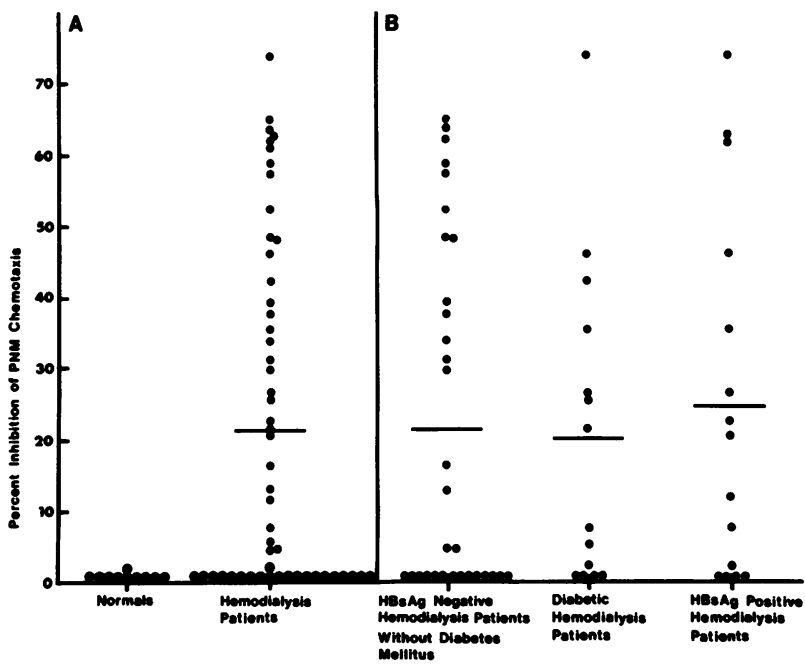

Figure 1 (A) Comparison of serum CIA in $53 \mathrm{HD}$ patients and 10 normal controls. (B) Comparison of serum CIA in nondiabetic, $\mathrm{HB}_{\mathrm{s}} \mathrm{Ag}$-negative $\mathrm{HD}$ patients with both diabetic $\mathrm{HD}$ patients, and $\mathrm{HB}_{\mathrm{s}} \mathrm{Ag}$-positive $\mathrm{HD}$ patients. The mean serum CIA in these three groups of patients were 21.5, 20.4 , and $24.7 \%$, respectively.

chemotactic response. When $10 \mathrm{HD}$ patients' sera with marked CIA were tested for spontaneous intrinsic serum chemotactic activity with a $10 \%$ concentration of fresh patient serum alone, chemotactic activity in these samples was markedly less than that found in five normal serum controls, $(51 \pm 28$ cells/HPF for HD patient sera vs. $116 \pm 35$ cells/HPF for normal serum controls). Furthermore, when four of these HD-patient sera were titrated by twofold dilutions from 10 to $1.25 \%$, intrinsic chemotactic activity decreased. Thus the possibility that the suppressed chemotactic response was caused by deactivation of PMN by excessive quantities of chemotactic factor in patient sera was unlikely.

Within the total group of $53 \mathrm{HD}$ patients there were 14 diabetics with a mean CIA of $20 \%, 5$ insulindependent diabetics with a mean CIA of $28 \%$, and 9 diabetics controlled without insulin with a mean CIA of $17 \%$. When 15 hepatitis B surface antigen $\left(\mathrm{HB}_{\mathrm{S}} \mathrm{Ag}\right.$ )-positive, HD patients (mean $\mathrm{CIA}, 25 \%$ ) were compared to 38 antigen-negative patients (mean CIA, $20 \%$ ), no significant difference was found. Fig. 1B separately compares nondiabetic, $\mathrm{HB}_{\mathrm{S}} \mathrm{Ag}$-negative $\mathrm{HD}$ patients to both diabetic $\mathrm{HD}$ patients and to $\mathrm{HB}_{\mathrm{S}} \mathrm{Ag}$ positive HD patients. No one disease subgroup could singularly account for the serum CIA seen in HD patients; chronic HD itself seemed to be the common denominator.

To study the effect of a single HD procedure on the presence of serum CIA, pre-HD sera from nine patients were compared to autologous post-HD samples. Although small changes in CIA were observed in individual patients, no significant difference by paired $t$ test analysis was observed between pre(mean CIA, 34\%) and post-HD (mean CIA, 34\%) samples. This suggested that a single HD procedure did not consistently increase or decrease CIA. Information regarding the number of months each patient had undergone HD was available for 49 of the $53 \mathrm{HD}$ patients. These were grouped with respect to duration of HD to see if multiple HD procedures might alter the serum CIA (Fig. 2). A significant difference $(P<0.05)$ by chi-square analysis was found between those 12 patients undergoing HD less than 3 mo (mean CIA, 1\%) and those 37 on HD for longer than 3 mo (mean CIA, 24\%). Sera from 21 of the latter 37 patients $(57 \%)$ displayed CIA, whereas sera from the 12 patients with $<3$ mo HD, failed to inhibit chemotaxis. 9 mo after the initial testing we resampled and tested the eight patients who initially had marked CIA and five patients who had initially been on HD $<3$ mo without demonstrable CIA (Fig. 3). We found that all sera from patients initially displaying CIA retained it, and that 4 of the 5 patients initially lacking CIA after $<3$ mo of $\mathrm{HD}$, had now acquired it. We then retested five surviving patients from the $>3$-mo groups, who, on initial testing, had no serum CIA. 18 mo after initial testing, all five still lacked serum CIA. Thus, after 3 mo of HD many patients developed CIA, whereas the five patients not developing the CIA by this time, did not do so at subsequent testing 18 mo later. From this data, it was assumed that patients lacking serum CIA after 3 mo of $\mathrm{HD}$ would be unlikely to aquire it and that HD patients with serum CIA would retain it. When $13 \mathrm{HD}$

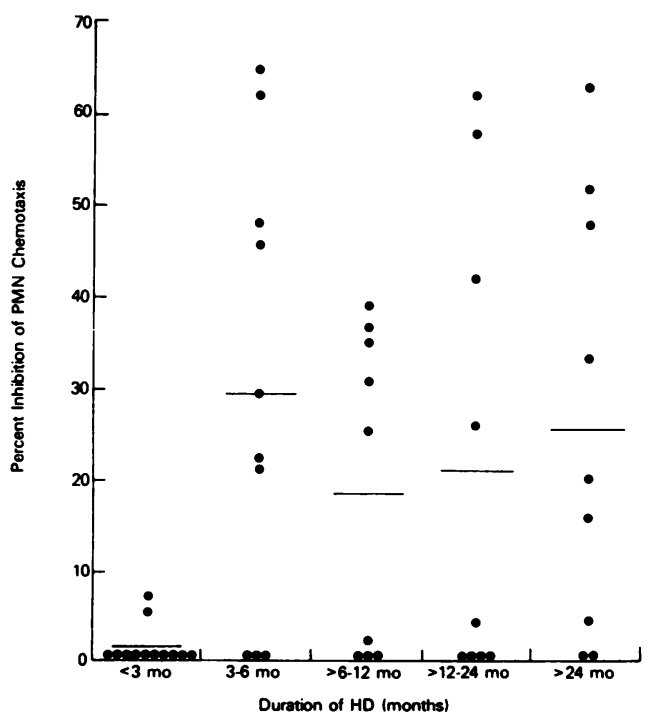

FIgURE 2 Relationship of serum CIA to duration of HD. HD patients with $<3$ mo HD showed significantly less serum CIA $(P<0.01)$ than did those with $>3$ mo HD. 


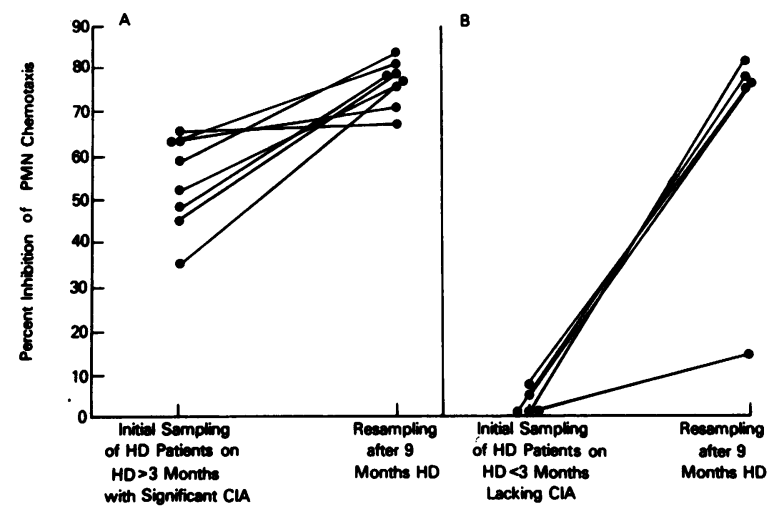

FIGURE 3 Sequential assays for serum CIA over a 9-mo interval of HD. (A) Tests on eight HD patients showing initial CIA and subsequent testing 9 mo later. Patients were on HD throughout the duration, and all eight patients retained serum CIA. (B) Results of studies on five HD patients with $<3$ mo of HD who initially lacked CIA, and subsequent studies 9 mo later. Four of the five patients acquired marked CIA.

patients lacking serum CIA after more than 3 mo of HD were compared to 19 HD patients with CIA, no significant difference in either infection or mortality was found between the two groups during the year after initial serum sampling. Patients receiving renal transplants were excluded from this analysis. 8 of the $13(61.5 \%)$ HD patients without CIA and 13 of the 19 (68.4\%) HD patients with CIA, suffered clinical, culture-documented infections including septicemia, vascular access site infections, soft tissue infections, as well as respiratory and urinary tract infections. 7 of the $13(53.8 \%)$ HD patients without CIA and 4 of the $19(21.1 \%)$ HD patients with CIA, died. Though the difference between mortality in the two groups was not statistically significant, a trend between the presence of serum CIA and a decreased mortality was evident, $P=0.0721$ with the Fishers exact test by two tailed analysis.

\section{Concentration dependence of serum CIA}

Six patient sera were titrated for concentration dependence of serum CIA with increasing amounts of test sera and a constant amount of CD chemotactic factor (Fig 4). As increasing amounts of patient sera were added, greater inhibition was observed.

\section{Neutralization of serum CIA with normal serum}

Each of three HD patients' sera with marked CIA (mean CIA, 66.9\%) were simultaneously assayed for CIA both with CD chemotactic fragment alone and with varying percentages of pooled normal sera from five normal subjects. The pooled normal sera was

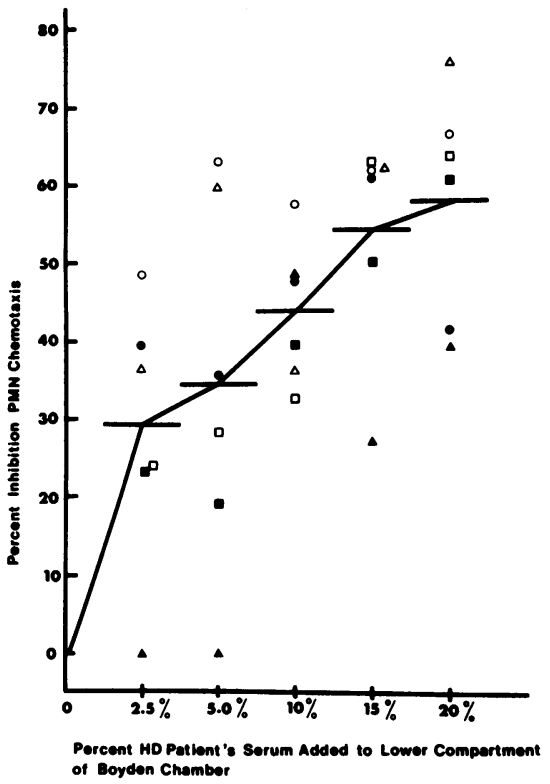

Figure 4 Concentration dependence of serum CIA. Each of six different symbols represents test results from a specific HD patient. The curve connects the mean serum CIA (horizontal lines) found at each percentage of HD patient's serum.

placed in the lower compartment of the Boyden chamber containing a constant quantity of CD chemotactic factor and 10\% HD patient serum. The results showed that as greater amounts of pooled normal sera were added, CIA decreased; but even when $20 \%$ pooled normal sera was added, $>20 \%$ inhibition remained in two of three patients tested (Fig. 5). Therefore, the CIA in HD patient sera could be partially counteracted by the addition of high concentrations of pooled normal serum.

\section{Partial characterization of serum CIA}

Effect of heat treatment. Sera from four patients with marked CIA (mean CIA, 56\%) were heat-treated at $56^{\circ} \mathrm{C}$ for $30 \mathrm{~min}$ and tested for CIA. The data indicate that the serum CIA in these patients was heat stable (postheat treatment mean CIA, 71\%).

Ammonium sulfate precipitation. Whole sera from four HD patients were salted out with 30 and $50 \%$ $\mathrm{NH}_{4} \mathrm{SO}_{4}$ (Fig. 6). At $30 \%$, all measurable CIA remained soluble, whereas at $50 \%$, significant CIA could be demonstrated in both soluble and precipitating fractions, with the majority still present in the soluble fraction. When supernatant fractions from the $30 \%$ $\mathrm{NH}_{4} \mathrm{SO}_{4}$ precipitation were heat-treated at $56^{\circ} \mathrm{C}$ for 45 min, no loss in CIA was observed. Thus, the CIA in all four patients' soluble fractions (mean CIA before heat treatment $=69 \%$ ) was heat stable (mean CIA after heat treatment $=79 \%$ ). 


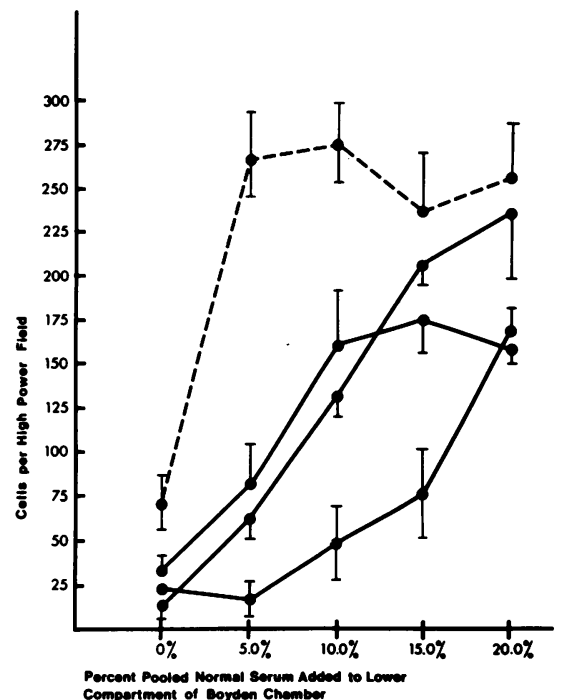

Figure 5 Neutralization of serum CIA with normal serum. Each of three HD patients' sera at a $10 \%$ concentration was assayed for CIA with varying amounts of pooled normal serum added to the lower compartment of the Boyden chamber together with a constant amount of CD chemotactic factor. Results are expressed in cells per HPF $\pm 1 \mathrm{SD}$. The dotted line (- - ) represents control results adding normal pooled sera to CD chemotactic factor in the absence of HD patients' sera. Each solid line (-) represents the results when this same titration was performed in the presence of a HD patient's serum.

\section{Separation of sera by sucrose density gradient centrifugation}

HD patient sera were separated by $5-20 \%$ sucrose density gradient centrifugation and each of the 15 fractions were tested for CIA with CD chemotactic factor as a chemotactic stimulus. The results of three patients' sera shown in Fig. 7 indicate a major peak of inhibition sedimenting slightly faster than the

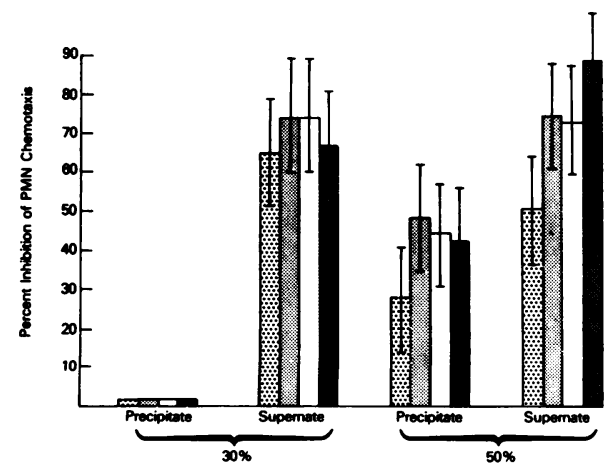

FIGURE 6 Fractionation of HD patient serum by ammonium sulfate precipitation. Data shows the presence of CIA in various fractions with four different patient sera and are expressed as mean inhibition $\pm 1 \mathrm{SD}$.

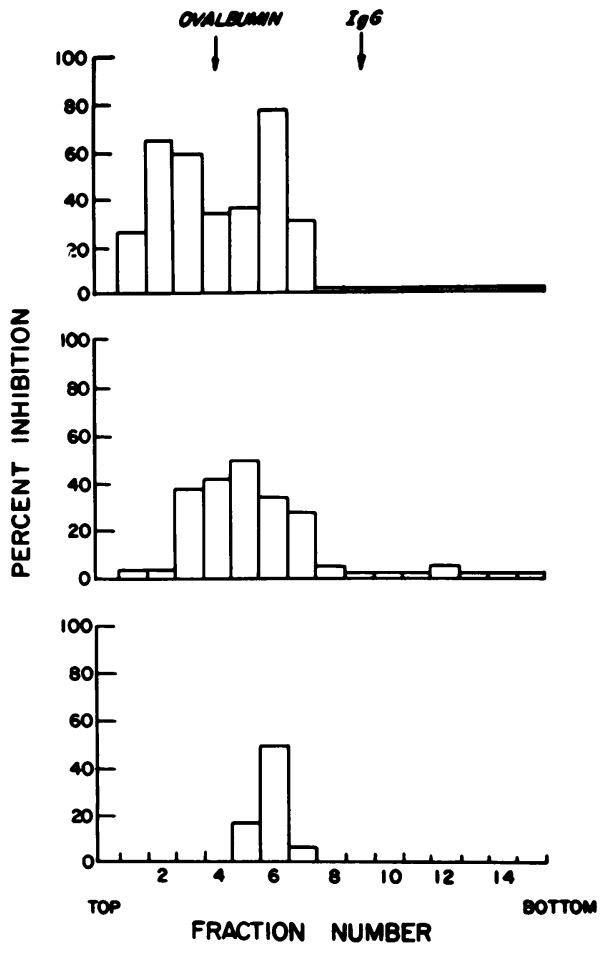

FIGURE 7 Separation of three HD patient sera by $5-20 \%$ sucrose density gradient centrifugation. The top of the gradient is on the left and the position of IgG and ovalbumin markers are shown. Data are expressed as percent inhibition of chemotaxis with each fraction with CD chemotactic factor as a control attractant.

ovalbumin marker, but slower than the IgG marker with a sedimentation coefficient of $\cong 4 \mathrm{~S}$. In some cases a slower sedimenting inhibitor was observed, an example of which is shown in the first sample in the same figure. This component was found only occasionally and appeared to be smaller than the $43,000 \mathrm{~mol}$ wt ovalbumin marker.

\section{CIA in serum fractions separated by size with ultrafiltration techniques}

Four normal and eight HD patients' sera were fractionated by ultrafiltration with membranes having pore sizes permitting passage of substances with mol wt of $<30,000$. Each sample was divided into two fractions of mol wt $>30,000$ and $<30,000$. The concentrated serum fractions were all corrected to their original volumes with phosphate-buffered saline and tested for CIA (Fig. 8). Significant differences of CIA were found between normal and HD patients' sera in the fractions of mol wt $>30,000(P<0.005)$. Substantial CIA was found in both serum fractions in at least some of the test sera (three of eight). Two of the three patients' sera studied by sucrose density centrifugation were also fractionated by ultrafiltration. When data 


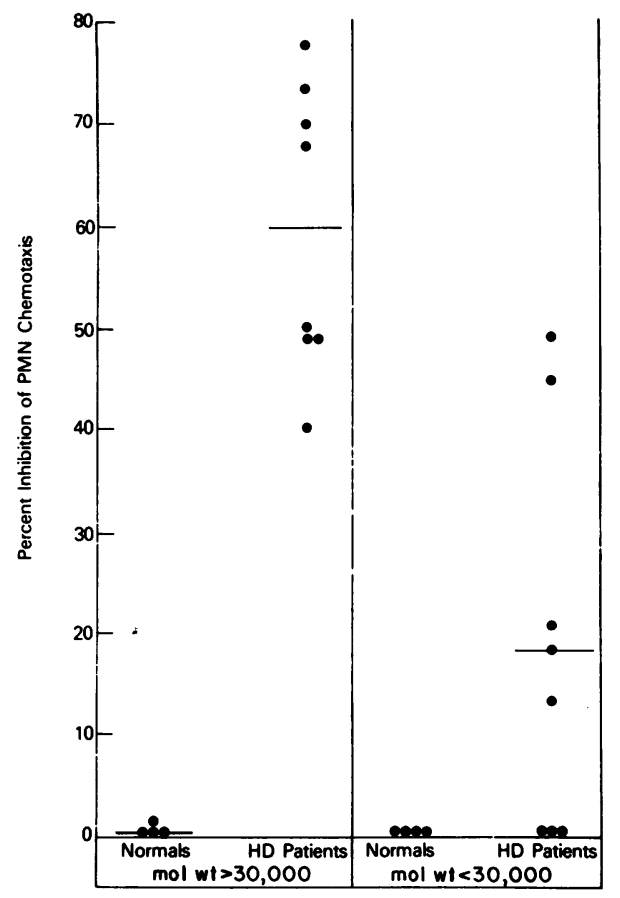

FIGURE 8 CIA in normal or HD patient sera after separation into fractions of $>30,000 \mathrm{~mol}$ wt and $<30,000 \mathrm{~mol}$ wt by ultrafiltration through an Amicon membrane. CIA was only found in patient sera, and was predominantly in the $>30,000 \mathrm{~mol}$ wt fractions that failed to pass through the membrane.

from samples separated by sucrose density gradient centrifugation (Fig. 7) were compared to ultrafiltration data (Fig. 8), the sample that contained the smaller inhibitor by sucrose density gradient centrifugation also showed some CIA in the serum fraction passing through the $30,000 \mathrm{~mol}$ wt ultrafiltration membrane. Conversely, the third sample showing only the $4 \mathrm{~S}$ inhibitor (Fig. 7) had CIA only in the $>30,000 \mathrm{~mol} \mathrm{wt}$ fraction by ultrafiltration.

\section{Preincubation of normal PMN with HD patients' sera}

Equal volumes of a normal PMN suspension $\left(7.0 \times 10^{6} \mathrm{PMN} / \mathrm{ml}\right)$ were centrifuged and the cell pellets resuspended in $10 \%$ solutions of 4 normal and $10 \mathrm{HD}$ patients' sera. The $10 \mathrm{HD}$ patients' sera were known to contain marked CIA ranging from 48 to $80 \%$ inhibition with a mean of $65 \pm 12 \%$. The cell-serum suspensions were incubated at $37^{\circ} \mathrm{C}$ for $1 \mathrm{~h}$, washed in HBSS three times, and resuspended to the original $7.0 \times 10^{6} \mathrm{cells} / \mathrm{ml}$. Cell suspensions were tested for chemotactic responsiveness to $\mathrm{CD}$ chemotactic factor along with control cells preincubated in HBSS alone. All cell preparations displayed equivalent chemotaxis indicating that HD patients' sera did not contain cell-directed inhibitors capable of irreversibly inhibiting PMN chemotaxis.

\section{Chemotactic factor specificity}

Each of $10 \mathrm{HD}$ patients' sera known to have marked serum CIA were tested with three different chemotactic factors (Fig. 9). The test sera were assayed with CD chemotactic factor, bacterial chemotactic factor, and casein as chemotactic stimulants. Control chemotaxis was $203 \pm 33,144 \pm 29$, and $120 \pm 18$ cells/HPF, respectively. The mean inhibition with $\mathrm{CD}$ chemotactic factor as the chemotactic stimulus was $61 \%$, whereas with bacterial factor and casein, 52 and $14 \%$ inhibition was observed, respectively. Thus, mean CIA was maximal with $C D$ chemotactic factor, slightly less with bacterial factor, and markedly less when casein was used. These data suggested that the CIA in patient sera is at least partially directed against the chemotactic factor because the presence of only cell-directed inhibitors might be expected to suppress migration equally well with all three factors.

\section{$C D$ chemotactic factor inactivation by $H D$ patients' serum fractions}

As previously indicated (Fig. 8) when HD patients' sera were fractionated, all fractions with mol wt $>30,000$, displayed marked CIA. These same fractions from normal and HD patients' sera, which contained molecules of $>30,000$ daltons, were incubated at a $10 \%$ final concentration with CD chemotactic factor for $1 \mathrm{~h}$ at $37^{\circ} \mathrm{C}$. The mixture was then forced through a 30,000-dalton pore size membrane, which permitted passage of CD chemotactic factor $(17,500 \mathrm{~mol} \mathrm{wt})$ but not the $>30,000 \mathrm{~mol}$ wt inhibitory substance. The

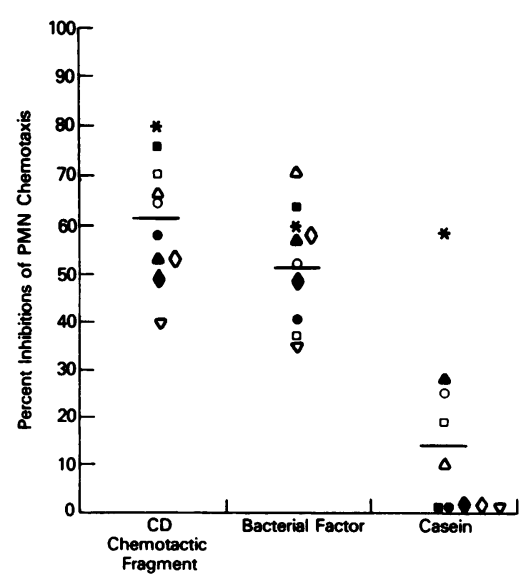

FIGURE 9 Effect of HD patient sera with various chemotactic factors. 
filtrates that passed through the membrane were collected and tested for chemotactic activity (Fig. 10). None of the six normal serum fractions reduced the chemotactic activity of the $\mathrm{CD}$ chemotactic factor by $>20 \%$ (mean reduction, $3 \%$ ), whereas, 8 of the $10 \mathrm{HD}$ patients' sera reduced its chemotactic activity by $>20 \%$ (mean reduction for all 10 samples, $43 \%$ ). This significant difference $(P<0.001)$ suggests that factors present in the HD patient serum fractions with mol wt $>30,000$ inactivate or complex with the CD chemotactic factor thereby reducing its chemotactic activity or ability to pass through the 30,000 -dalton pore size membrane.

\section{DISCUSSION}

The sera from 24 of $35 \mathrm{HD}$ patients were found to inhibit normal PMN chemotaxis by $>20 \%$. Serum CIA was not altered by a single HD procedure and in the patients studied here, appeared only after 3 mo of HD. Titration experiments indicated that inhibition was concentration dependent. CIA was heat stable, and remained soluble after $30 \%$ ammonium sulfate precipitation, and was only partially precipitated with $50 \%$ ammonium sulfate. Sucrose density gradient

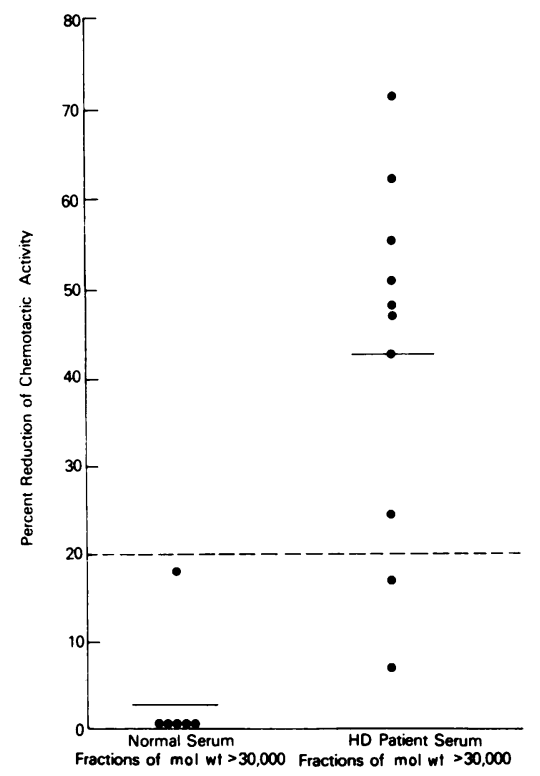

FIGURE 10 Demonstration of chemotactic factor directed inhibitors in HD patient serum. In each case the $>30,000$ mol wt fraction was preincubated with CD chemotactic factor and the mixture then put through the filtration membrane, which retains the serum inhibitors, but not chemotactic factor. Data are expressed as percent reduction of the chemotactic activity found in the filtrate which passed through the membrane when compared to a similarly treated preparation of $\mathrm{CD}$ chemotactic factor in the absence of serum fractions. centrifugation indicated that the major inhibitory activity was concentrated in a region with a molecular weight slightly greater than ovalbumin.

The CIA found in whole serum appeared factor directed because chemotactic inhibition was greater with some chemotactic factors than with others (Fig. 9). Cell-directed inhibitors such as those previously described in patients with IgA myeloma (16) have been shown to suppress the chemotactic response to all three chemotactic factors used in this study. Likewise, several other studies have demonstrated that patients with multiple chemotactic inhibitors, including celldirected inhibitors, suppress neutrophil chemotaxis to all chemotactic factors (18-21). In addition, preincubation of normal PMN with inhibitory sera failed to depress cellular chemotaxis. This supports a factordirected inhibitor though not excluding a rapidly reversible, cell-directed inhibitory process. A chemotactic factor-directed inhibitor was further upheld by demonstrating that the $>30,000 \mathrm{~mol}$ wt serum fraction directly interacted with $C D$ chemotactic factor as shown in the experiments in Fig. 9. Although these experiments imply inactivation of CD chemotactic factor by HD patient sera, they do not exclude a possible complexing of the inhibitor with chemotactic factor, thereby preventing its passage through the PM30 membrane.

Several previously described, factor-directed inhibitors share some of the properties of the CIA described here. Other investigators have described a chemotactic factor inactivator present in low levels in normal serum $(22,23)$ and supernormal levels in certain pathological conditions $(24,25)$. Chemotactic factor inactivator remains soluble when treated with $45 \%$ ammonium sulfate, has a broad spectrum of activity including $\mathrm{C} 5$ chemotactic fragment and bacterial factor, and is heterogeneous with at least two components having sedimentation velocities of $4 \mathrm{~S}$ and $7 \mathrm{~S}$. The $4 \mathrm{~S}$ chemotactic factor inactivator inactivates C5a and resembles the CIA described in this study, with the possible exception of heat lability. Brozna et al. (26) have described two granulocyte lysosomal proteases, elastase and cathepsin $\mathrm{G}$, that inactivated both C3 and C5 chemotactic factors. Other investigators $(27,28)$ have reported human leukocyte elastases to range in mol wt from 22,000 to 33,000 . It is possible that these enzymes might contribute to the CIA described here. Wright and Gallin $(29,30)$ have described chemotactic factor inactivators released from the azurophilic granules of neutrophils after phagocytosis. They found at least two inactivators with mol wt 65,000 and 30,000 , and possibly one with a 10,000-12,000 mol wt. These factors inactivated C5 chemotactic fragment and had molecular weights consistent with the inhibitors described here. In 
contrast, their factors were reported to be heat labile. More recently, Perez et al. (31) have reported a serum inhibitor of C5-derived chemotactic activity in patients with systemic lupus erythematosis. Chromatography of patient serum on Sephadex G-200 yielded three distinct inhibitor peaks. One inhibitory peak was heat stable at $56^{\circ} \mathrm{C}$ for $30 \mathrm{~min}$ and had an apparent mol wt of $50,000-60,000$, which is similar to the chemotactic inhibitor described here.

Several possible mechanisms might explain the appearance of this serum CIA in HD patients. It has long been recognized that renal disease itself can be associated with abnormal chemotaxis. In 1967, Gewurz et al. (32) noted a correlation between hypocomplementemia associated with glomerulonephritis and depressed neutrophil chemotaxis. In another study, Clark et al. (11) reported defective serum generation of chemotactic factors in rabbits with cephaloridineinduced acute renal failure. Salent et al. (12) have found that the chemotactic activity of endotoxinactivated sera from chronic renal insufficiency and HD patients was often decreased. This serum defect has not been upheld by several other studies (13-15).

Our data showing that patients undergoing more than 3 mo of HD have an increased incidence of CIA, strongly suggests that $\mathrm{HD}$ itself might contribute to the presence of CIA. Other immunological changes have been associated with the HD procedure including the generation of a plasma factor that augments granulocyte adherence $(10,33)$, complement-mediated pulmonary leukostasis (34-37), the presence of circulating free DNA (38), antinuclear antibodies (39), and anti-blood group N-like antibodies (40-42). Furthermore, cellulose membranes of the same type used in HD machinery have been shown to impair PMN random mobility (8).

Studies have shown that granulocyte destruction occurs with granulocyte-HD membrane interaction (43), and that release of PMN intracellular organelles can occur (44). Using phase-contrast microscopy, PMN from patients with renal failure have been shown to have more prominent cytoplasmic granules (45) and are reported to have increased intracellular cathepsin D (46). Already mentioned in this discussion, several intracellular substances released from granulocytes are known to inactivate $C D$ chemotactic factors $(26,29,30)$. It is possible that granulocytes altered or destroyed by the HD process might release one or more of these factors.

Another granulocyte released factor, the neutrophilimmobilizing factor described by Goetzl and Austen (47), is a heat stabile, cell-directed, irreversible inhibitor of cellular chemotaxis and random mobility with a mol wt of $\cong 5,000$. In our study, normal cells preincubated with HD patients' sera displayed normal chemotactic response, making our CIA incompatible with the neutrophil-immobilizing factor.

Together with possible increased accumulation and(or) increased release of PMN intracellular inhibitory substances, an increased survival for such substances might be contributory to the CIA seen in HD patients. In this study, the greatest and most consistent CIA was demonstrated in the serum fraction of mol wt $>30,000$. Though a molecule of this size might be easily cleared by normally functioning renal glomeruli, the pore size of the standard HD membrane permits passage of molecules with mol wt of up to $10,000-12,000$. Thus, any inhibitory substance of mol wt $>30,000$ might exert a prolonged and intensified effect in this group of patients. An alternative theory is that the presence of circulating chemotactic inhibitors may be a host response to chemotactic factors generated by the complement activation known to occur during HD $(30,32-34)$. In this case, the factor-directed inhibitors could protect the host from adverse effects of excessive C5-fragment generation. Consistent with this hypothesis is the finding of a similar inhibitor in systemic lupus erythematosis (31), another condition also associated with chronic complement activation and therefore C5 fragment generation.

Abnormal chemotaxis has been associated with nondialyzed chronic renal insufficiency $(11,12)$. Because of this, similar defects found in HD patients might have also been ascribed to their impaired renal function. Several investigators have examined PMN chemotaxis among HD patients (12-15). Three of these studies that failed to find a serum defect (13-15) used a different chemotactic stimulus than was utilized in our assay system. Baum et al. (13) activated whole serum with endotoxin, probably generating multiple chemotactic factors, some of which may not have been inhibited by the $4 \mathrm{~S}$ factor-directed inhibitor. Greene et al. (14) used plasma in place of serum and $E$. coli bacterial factor instead of CD chemotactic factor. The CIA described in our study was slightly less inhibitory against bacterial factor-promoted chemotaxis than it was when CD chemotactic factor was used. These same investigators only tested a total of four HD patients for the presence of a circulating inhibitor and may have easily overlooked such an inhibitor as it was found in less than half of our patients. Bjorksten et al. (15) tested $11 \mathrm{HD}$ patients for the presence of a circulating chemotactic inhibitor. They compared cellular chemotaxis of normal PMN suspended in normal serum to PMN suspended in HD patients' plasma. The PMN suspended in HD patient plasma showed a slightly reduced mean chemotactic index though this reduction was not found to be significant. This mean reduction of chemotaxis was similar to our results although in our studies 
the patient serum was placed in the same compartment as the chemotactic factor.

Several investigators have recognized infection as a leading cause of death among chronic HD patients $(3,4)$. We could not correlate the presence of serum CIA with a significant increase in either infection or mortality in 32 of our HD patients studied over a 12-mo period, although a trend $(P=0.0721)$ between the presence of CIA and decreased mortality was apparent. Additional studies of larger numbers of patients over a more extended period of time are necessary to substantiate the presence or absence of correlation between serum CIA and infectious and(or) fatal complications among HD patients.

Although no significant increase in infection was noted in patients with CIA as opposed to patients without CIA, it is feasible that some of the altered host responses noted in $\mathrm{HD}$ patients might be related to the presence of serum CIA. For instance, HD patients have been shown to display cutaneous anergy (13) in the face of improved in vitro lymphocyte function after HD (48-50). Several previous studies have shown serum inhibitors of chemotaxis to be associated with cutaneous anergy $(18,21)$. As suppression of cell-mediated immunity is crucial to kidney transplant survival, it is possible that serum CIA could influence the clinical course of kidney transplantation among previously hemodialyzed recipients (51). Further studies will be essential to determine if this chemotactic inhibition like those previously described is associated with anergy, or involved in kidney transplant survival. Another common clinical situation that challenges HD patients is the respiratory distress associated with complement-mediated pulmonary leukostasis (34-37). Studies indicate that C5a is responsible for $\mathrm{HD}$-associated pulmonary leukostasis (37). It is therefore conceivable that serum CIA might play a protective role and block C5a to prevent complement-mediated pulmonary leukostasis. That CIA might be beneficial to the host is suggested by the statistical trend found in our group of patients but remains speculative at this time.

We conclude that many patients undergoing maintenance HD for a duration of 3 mo or more acquire serum CIA. The inhibitor consists of a major $4 \mathrm{~S}$ heatstable substance directed at $\mathrm{CD}$ chemotactic factor. This inhibitor resembles the factor-directed inhibitor found in patients with systemic lupus erythematosis (31). It is proposed that this inhibitor may be released from granulocytes during HD or be a host response to control C5a generation associated with HD.

\section{ACKNOWLEDGMENTS}

We are indebted to Dr. Dale Erickson for contributing patients to the study and to the Hemodialysis Unit personnel at the
Veteran's Administration and Presbyterian Hospitals and Bernalillo County Medical Center for their cooperation with patient scheduling and blood sampling. We thank Dr. D. Pathak for statistical analysis, and R. Spangler for laboratory assistance.

This work was supported by grants CA 20819 and AI 13433 from the U. S. Public Health Service.

\section{REFERENCES}

1. Balch, H. H. 1955. The effect of severe battle injury and of post-traumatic renal failure on resistance to infection. Ann. Surg. 142: 145-163.

2. Montgomerie, J. Z., G. M. Kalmanson, and L. B. Guze. 1968. Renal failure and infection. Medicine (Baltimore). 47: 1-32.

3. Burton, B. T., K. K. Krueger, and F. A. Bryan, Jr. 1971. National registry of long-term dialysis patients. JAMA (J. Am. Med. Assoc.) 218: 718-722.

4. Lowrie, E. G., J. M. Lazarus, A. J. Mocelin, G. L. Bailey, C. L. Hampers, R. E. Wilson, and J. P. Merrill. 1973. Survival of patients undergoing chronic hemodialysis and renal transplantation. N. Engl. J. Med. 288: 863-867.

5. Davidson, W. D., and K. R. Tanaka. 1971. Effect of uremia on phagocytosis-stimulated glucose oxidation in human granulocytes. Clin. Res. 19: 416.

6. Montgomerie, J. Z., G. M. Kalmanson, and L. B. Guze. 1972. Leukocyte phagocytosis and serum bactericidial activity in chronic renal failure. Am. J. Med. Sci. 264: 385-393.

7. Burleson, R. L. 1973. Reversible inhibition of phagocytosis in anephric uremic patients. Surg. Forum. XXIV: 75-77.

8. Henderson, L. W., M. E. Miller, R. W. Hamilton, and M. E. Norman. 1975. Hemodialysis leukopenia and polymorph random mobility - a possible correlation. J. Lab. Clin. Med. 85: 191-197.

9. Ruley, E. J., S. W. Huang, J. Plant, and N. Morris. 1976. Defective phagocyte adherence in acute post streptococcal glomerulonephritis: clinical and laboratory observations. J. Pediatr. 89: 748-754.

10. MacGregor, R. R. 1977. Granulocyte adherence changes induced by hemodialysis, endotoxin, epinephrine, and glucocorticoids. Ann. Intern. Med. 86: 35-39.

11. Clark, R. A., B. H. Harmory, G. H. Ford, and H. R. Kimball. 1972. Chemotaxis in acute renal failure. J. Infect. Dis. 126: $460-463$.

12. Salant, D. J., A. Glover, R. Anderson, A. M. Meyers, R. Rabkin, J. A. Myburgh, and A. R. Rabson. 1976. Depressed neutrophil chemotaxis in patients with chronic renal failure and after renal transplantation. J. Lab. Clin. Med. 88: 536-545.

13. Baum, J., R. V. M. Cestero, and R. B. Freeman. 1975. Chemotaxis of the polymorphonuclear leukocyte and delayed hypersensitivity in Uremia. Kidney Int. Suppl. 2:S-147-S-153.

14. Greene, W. H., C. Ray, S. M. Maner, and P. G. Quie, 1976. The effect of hemodialysis on neutrophil chemotactic responsiveness. J. Lab. Clin. Med. 88: 971-974.

15. Bjorksten, B., S. M. Mauer, E. L. Mills, and P. G. Quie. 1978. The effect of hemodialysis on neutrophil chemotactic responsiveness. Acta. Med. Scand. 203: 67-70.

16. Van Epps, D. E., and R. C. Williams, Jr. 1976. Suppression of leukocyte chemotaxis by Human Iga Myeloma Components. J. Exp. Med. 144: 1227-1242.

17. Vallota, E. H., and H. J. Muller-Eberhard. 1973. Formation of C3a and C5a anaphylatoxins in whole human serum 
after inhibition of the anaphylatoxin inactivator. J. Exp. Med. 137: 1109-1123.

18. Van Epps, D. E., D. L. Palmer, and R. C. Williams, Jr. 1974. Characterization of serum inhibitors of neutrophil chemotaxis associated with anergy. J. Immunol. 113: 189-200.

19. Van Epps, D. E., R. G. Strickland, and R. C. Williams, Jr. 1975. Inhibitors of leukocyte chemotaxis in alcohol liver disease. Am. J. Med. 59: 200-207.

20. Van Epps, D. E., and R. C. Williams, Jr. 1976. Serum chemotactic inhibitory activity: heat activation of chemotactic inhibition. Infect. Immun. 13: 741-749.

21. Van Epps, D. E., and R. C. Williams, Jr. 1978. Leukocyte Chemotaxis. Raven Press, New York. 237-253.

22. Berenberg, J. L., and P. A. Ward. 1973. Chemotactic Factor Inactivator in Normal Human Serum. J. Clin. Invest. 52: 1200-1206.

23. Till, G., and P. A. Ward. 1975. Two distinct chemotactic factor inactivators in human serum. J. Immunol. 114: 843-847.

24. Ward, P. A., and J. L. Berenberg. 1974. Defective regulation of inflammatory mediators in Hodgkin's disease. Supernormal levels of chemotactic factor inactivator. N. Engl. J. Med. 290: 76-80.

25. Ward, P. A., S. Goralnick, and W. E. Bullock. 1976. Defective leukotaxis in patients with lepromatous leprosy. J. Lab. Clin. Med. 87: 1025-1032.

26. Bronza, J. P., R. M. Senior, D. L. Kreutzer, and P. A. Ward. 1977. Chemotactic factor inactivators of human granulocytes. J. Clin. Invest. 60: 1280-1288.

27. Taylor, J. C., and I. P. Crawford. 1975. Purification and preliminary characterization of human leukocyte elastase. Arch. Biochem. Biophys. 169: 91-101.

28. Baugh, R. J., and J. Travis. 1976. Human leukocyte granule elastase: rapid isolation and characterization. Biochemistry. 15: 836-841.

29. Wright, D. G., and J. I. Gallin. 1975. Modulation of the inflammatory response by products released from human PMN leukocytes during phagocytosis. Generation and inactivation of chemotactic factor C5a. Inflammation. 1: 23-39.

30. Wright, D. G., and J. I. Gallin. 1977. A functional differentiation of human neutrophil granules: generation of C5a by a specific (secondary) granule product and inactivation of $\mathrm{C} 5 \mathrm{a}$ by azurophil (primary) granule products. J. Immunol. 119: 1068-1076.

31. Perez, H. D., M. Lipton, and I. M. Goldstein. 1978. A specific inhibitor of complement (C5)-derived chemotactic activity in serum from patients with systemic lupus erythematosis. J. Clin. Invest. 62: 29-38.

32. Gewurz, H., A. R. Page, R. J. Pickering, and R. A. Good. 1967. Complement activity and inflammatory neutrophil exudation in man. Int. Arch. Allergy Appl. Immunol. 32: 64-90.

33. Guerrero, I. C., A. D. Schreiber, and R. R. Macgregor. 1977. Granulocytopenic Factor in Hemodialysis. Seventh Interscience Conference on Antimicrobial Agents and Chemotherapy. Abstract 217.

34. Craddock, P., J. Fehr, K. L. Brigham, and H. Jacob. 1975. Pulmonary capillary leukostasis: a complement $\left(\mathbf{C}^{\prime}\right)$ mediated complication of hemodialysis. Clin. Res. 23: 402A. (Abstr.)

35. Craddock, P. R., J. Fehr, K. L. Brigham, R. S. Kronenberg, and H. S. Jacob. 1977. Complement and leukocytemediated pulmonary dysfunction in hemodialysis. $N$. Engl. J. Med. 296: 769-774.

36. Craddock, P. R., J. Fehr, A. P. Dalmasso, K. L. Brigham, and H. S. Jacob. 1977. Hemodialysis leukopenia, pulmonary vascular leukostasis resulting from complement activation by dialyzer cellophane membranes. J. Clin. Invest. 59: 879-888.

37. Craddock, P. R., D. Hammerschmidt, J. G. White, A. P. Dalmasso, and H. S. Jacob. 1977. Complement (C5a)induced granulocyte aggregation in vitro, a possible mechanism of complement mediated leukostasis and leukopenia. J. Clin. Invest. 60: 260-264.

38. Steinman, C. R., and A. Ackard. 1977. Appearance of circulating DNA during hemodialysis. Am. J. Med. 62 : 693-697.

39. Nolph, K. D., F. C. Husted, G. C. Sharp, and A. W. Siemsen. 1976. Antibodies to nuclear antigens in patients undergoing long term hemodialysis. Am. J. Med. 60: 673-676.

40. Howell, E. D., and H. A. Perkins. 1972. Anti-N-like antibodies in the sera of patients undergoing chronic hemodialysis. Vox Sang. 23: 291-299.

41. McLeish, W. A., A. F. Brathwaite, and P. M. Peterson. 1975. Anti-N-antibodies in hemodialysis patients. Transfusion (Phila). 15: 43-45.

42. Kaehny, W. D., G. E. Miller, and W. L. White. 1977. Relationship between dialyzer reuse and the presence of anti-N-like antibodies in chronic hemodialysis patients. Kidney Int. 12: 59-65.

43. Buscarini, L., and F. Bassi. 1972. Leukocyte loss in haemodialysis. Acta Haematol. (Basel). 48: 278-282.

44. Marshall, J. W., D. J. Ahearn, F. J. Nothum, J. Esterly, K. D. Nolph, and J. F. Matter. 1974. Adherence of blood components to dialyzer membranes morphological studies. Nephron. 12: 157-170.

45. Brogen, T. D. 1967. Phagocytosis by polymorphonuclear leukocytes from patients with renal failure. $\mathrm{Br}$. Med. J. 3: 569-599.

46. Guckian, J. C., L. R. Karrh, J. L. Copeland, and J. McCoy. 1971. Phagocytosis by polymorphonuclear leukocytes in patients with renal failure on chronic hemodialysis. Tex. Rep. Biol. Med. 29: 193-198.

47. Goetzl, E. J., and K. F. Austen. 1972. A neutrophilimmobilizing factor derived from human leukocytes. I. Generation and partial characterization. J. Exp. Med. 136: $1564-1580$.

48. Newberry, W. M., and J. P. Sanford. 1971. Defective cellular immunity in renal failure: depression of reactivity of lymphocytes to phytohemagglutinin by renal failure serum. J. Clin. Invest. 50: 1262-1271.

49. Webel, M. L., R. E. Ritts, W. A. Briggs, and J. A. Light. 1976. Lymphocyte blastogenesis in patients receiving hemodialysis. Arch. Intern. Med. 136: 682-687.

50. Hoy, W. E., R. V. M. Cestero, and R. B. Freeman. 1978. Deficiency of $T$ and $B$ lymphocytes in uremic subjects and partial improvement with maintenance hemodialysis. Nephron. 20: 182-188.

51. Wilson, W. E. C., C. H. Kirkpatrick, and D. W. Talmage. 1964. Immunologic studies in human organ transplantation. III. The relationship of delayed cutaneous hypersensitivity to the onset of attempted kidney allograft rejection. J. Clin. Invest. 43: 1881-1891. 